\title{
Télescope
}

Revue d'analyse comparée en administration publique

\section{Audit interne et contrôle de gestion : pour une meilleure collaboration, de Jacques Renard et Sophie Nussbaumer, Paris, Eyrolles Éditions d'Organisation, 2011, 232 p.}

\section{Youssef Slimani}

Volume 18, numéro 3, automne 2012

URI : https://id.erudit.org/iderudit/1013784ar

DOI : https://doi.org/10.7202/1013784ar

Aller au sommaire du numéro

Éditeur(s)

L'Observatoire de l'administration publique

ISSN

1203-3294 (imprimé)

1929-3348 (numérique)

Découvrir la revue

Citer ce compte rendu

Slimani, Y. (2012). Compte rendu de [Audit interne et contrôle de gestion : pour une meilleure collaboration, de Jacques Renard et Sophie Nussbaumer, Paris, Eyrolles Éditions d'Organisation, 2011, 232 p.] Télescope, 18(3), 186-188.

https://doi.org/10.7202/1013784ar d'utilisation que vous pouvez consulter en ligne.

https://apropos.erudit.org/fr/usagers/politique-dutilisation/ 


\section{AUDIT INTERNE ET CONTRÔLE DE GESTION : POUR UNE MEILLEURE COLLABORATION}

De Jacques Renard et Sophie Nussbaumer, Paris, Eyrolles Éditions d'Organisation, 2011, 232 p.

A udit interne et contrôle de gestion : pour une meilleure collaboration est l'occasion d'un regard croisé d'un auditeur interne, Jacques Renard, et d'un contrôleur de gestion, Sophie Nussbaumer, sur les interactions entre ces deux fonctions au sein des organisations françaises. Les auteurs participent également, à des degrés différents, à la formation spécialisée en audit interne et en contrôle de gestion. Ils plaident pour un rapprochement des deux professions et pour une meilleure collaboration entre les deux fonctions dans le but d'améliorer la gouvernance des entreprises.

Le livre est divisé en trois parties principales. À la suite de l'introduction dans laquelle il est question de l'évolution historique des deux fonctions et de leurs définitions, la première partie note leurs convergences et précise leurs spécificités. L'audit interne et le contrôle de gestion sont ainsi deux fonctions essentielles qui contribuent, par des voies différentes, à la réalisation des objectifs de l'entreprise. Le contrôle de gestion assure le suivi des réalisations et l'élaboration de prévisions à long terme, tandis que l'audit interne détecte les risques et formule des propositions pour les éviter. Les auteurs indiquent que ces deux fonctions de conseil au management agissent dans des périmètres semblables en investissant toutes les activités d'une organisation. Deux types de convergences sont ensuite distinguées : celles relevant de l'assistance mutuelle et celles liées au domaine des relations internes. L'assistance mutuelle se traduit par la mise au service de chaque technique et de chaque procédé de l'autre fonction. Ainsi, l'audit interne examine, selon une approche par les risques, la fonction du contrôle de gestion et détermine dans quelle mesure elle répond aux attentes de l'organisation. Inversement, le contrôle de gestion veille à ce que la conception fonction de l'audit interne soit conforme à la stratégie de l'organisation. Les deux fonctions présentent aussi des similitudes dans la nature des liens relationnels avec les différents organes de l'entreprise qui se traduisent par l'importance du responsable de la fonction dans son influence et son autorité, ainsi que par un dialogue renforcé avec les responsables opérationnels. Les auteurs soulignent que l'évolution des deux fonctions permet de présenter leurs particularités comme des occasions à saisir pour une collaboration étroite dans le but d'une meilleure efficacité. Dans ce cadre, les risques ciblés par l'auditeur interne constituent une information primordiale pour le contrôleur de gestion et l'information centralisée par ce dernier alimente les démarches de l'auditeur interne.

La section suivante propose une feuille de route pour une meilleure organisation du travail et la création d'une synergie relationnelle au sein des organisations. Les auteurs notent que les nombreuses tentatives pour redéployer le contrôle de gestion et l'audit interne n'ont pas apporté la cohérence voulue. Pour remédier 
à cette situation, les auteurs préconisent une amélioration de la convergence des deux fonctions par l'entremise d'une concertation organisée qui résulte de plusieurs actions : amélioration dans la définition des périmètres, optimisation de la gestion, meilleure coordination des objectifs, harmonisation des finalités, adaptation des moyens et complémentarité organisée des méthodes et des outils. L'amélioration de la convergence doit être accompagnée par une totale adhésion des acteurs. Les auteurs appellent à une coordination des activités grâce à une participation concertée et organisée de tous les acteurs au contrôle interne. Ces derniers devraient aussi se limiter à leur propre champ d'expertise pour assurer une complémentarité de leurs actions. Il s'agit d'une démarche collective visant l'amélioration de la gouvernance. Selon Renard et Nussbaumer, la mise en place de ces nouvelles méthodes de travail doit être soutenue par une formation adéquate des différents acteurs.

Dans la troisième partie du livre, les auteurs proposent une autre organisation de travail qui favorise une meilleure concertation entre les deux fonctions. Ils recommandent de les regrouper, tout en conservant leurs spécificités, au sein d'une même structure rattachée à la direction générale et pilotée par une autorité commune maîtrisant les deux expertises. Le partage cohérent des tâches entre les deux activités devrait être garanti par une charte commune élaborée par le responsable de cette structure. Les auteurs estiment que ce type d'organisation permet à l'audit interne et au contrôle de gestion de devenir des acteurs efficaces de la gouvernance de l'entreprise. Cette organisation n'est pas nouvelle; elle est déjà initiée dans le monde anglo-saxon. Les auteurs soutiennent qu'elle contribue à une meilleure gouvernance, et ce, de trois façons différentes. En premier lieu, le fait d'optimiser la relation entre la poursuite de la performance et la gestion des risques permet de limiter la volatilité des résultats, d'améliorer l'allocation des ressources, de renforcer la planification stratégique et d'accroître la valeur ajoutée. En second lieu, le type de structure proposée favorise la coordination de la déontologie et des normes professionnelles des deux fonctions, dans une perspective de cohérence ou de complémentarité. Enfin, la concertation entre les deux fonctions facilite une harmonisation de l'assistance du management qui se concrétise sur cinq plans : renforcer l'alerte, mieux gérer les imprévus, rendre plus crédible la vision stratégique, améliorer l'information et conforter le développement durable, pilier d'une meilleure gouvernance.

En guise de conclusion, les auteurs abordent les problèmes actuels auxquels font face les organisations. Il s'agit d'un monde de travail qui évolue dans un environnement de plus en plus complexe. La concertation est défaillante en raison d'une organisation de travail trop segmentée, et elle est aggravée par des structures inadaptées qui nuisent à la bonne gouvernance. En plus des solutions proposées dans les chapitres du livre, à savoir des structures adaptées, un système d'information efficace pour les acteurs des deux fonctions et une direction générale bien informée, les auteurs avancent d'autres points qui s'articulent autour de la qualité de l'environnement de contrôle, la rationalité de l'organisation et le professionnalisme des acteurs. 
Le livre s'adresse en priorité aux gestionnaires d'entreprise et au milieu professionnel des fonctions de l'audit interne et du contrôle de gestion. La bibliographie se limite essentiellement à des monographies publiées en français et traitant de façon générale des deux fonctions. La démarche des auteurs d'amorcer un rapprochement entre les deux professions s'inscrit dans le contexte français et ne peut être généralisée que dans des cadres de gestion similaires. Certes, ils présentent un plaidoyer pour la convergence des deux fonctions, mais il apparaît que leur grand souci est de préserver leurs spécificités et leur champ d'action respectif. Par ailleurs, les pistes d'amélioration proposées restent dans les généralités et sont valables, sauf quelques exceptions, pour organiser les relations entre toutes les autres unités d'une organisation. Les auteurs auraient dû appuyer leur proposition par des données empiriques et citer des études qui mettent en évidence les avantages occasionnés par l'adoption d'une nouvelle structure regroupant l'audit interne et le contrôle de gestion.

Par YOUSSEF SLIMANI

Agent de recherche, Centre de recherche et d'expertise en évaluation (CREXE), École nationale d'administration publique 